\title{
"EL COMPROMISO DE LA UNIVERSIDAD FRENTE A UN MUNDO INCIERTO Y COMPLEJO: PROPUESTAS PARA LA FORMACIÓN DE PROFESIONALES REFLEXIVOS"
}

\begin{abstract}
La patología moderna del espíritu está en la hiper-simplificación que ciega la complejidad de lo real. (...) Sólo el pensamiento complejo nos permitirá civilizar nuestro conocimiento (MORIN, 2008, p.35).
\end{abstract}

\begin{abstract}
Resumen: En este este artículo se expone que una de las responsabilidades de la universidad en estos momentos es la formación profesionales reflexivos y críticos que puedan trabajar en entornos de complejidad. Se describen las características de los entornos complejos y a continuación se exponen las repercusiones que este planteamiento tiene en la manera de diseñar la formación de profesionales. Finalmente se describen las redes socioeducativas como forma de concretar las estrategias de trabajo en el territorio.
\end{abstract}

Palabras clave: Pensamiento complejo; Profesional reflexivo; Compromiso

La velocidad y la intensidad de las transformaciones sociales en el cambio de siglo están obligando a la universidad a reflexionar nuevamente sobre la mejor manera de asumir sus responsabilidades frente a la sociedad de la que forma parte. Partiremos de la idea clásica que ya indicaba Ortega y Gasset que "la Universidad tiene que estar abierta a la plena actualidad; más aún, tiene que estar en medio de ella, sumergida en ella" (1982, p.77) o, como años más tarde la formulaba Bricall (2000, p.34): "la Universidad no se debe contentar con adaptarse de forma pasiva a esta evolución social, sino que debe tomar parte activa en la misma en estrecho diálogo y cooperación con las demás entidades sociales, económicas, culturales y políticas".

También insistía en ello la Declaración Mundial sobre la Educación Superior en el Siglo XXI (UNESCO, 1998, p.5), cuando en su artículo 2 indicaba que la Universidad debe "poder opinar sobre los problemas éticos, 


$$
\begin{gathered}
\text { Jesús Vilar Martín } \\
\text { Gisela Riberas Bargalló } \\
\text { Genoveva Rosa Gregori } \\
\text { Facultad de Educación Social y Trabajo Social Pere Tarrés } \\
\text { Universidad Ramon Llull } \\
\text { España }
\end{gathered}
$$

culturales y sociales, con total autonomía y plena responsabilidad, por estar provistos de una especie de autoridad intelectual que la sociedad necesita para ayudarla a reflexionar, comprender y actuar".

Pero, ¿cómo traducir hoy esta idea de formar parte activamente, en qué concretarla? El proceso de creación del Espacio Europeo de Educación Superior (EEES) abrió un intenso debate sobre la esencia del pensamiento universitario frente a la amenaza de la mercantilización del conocimiento ante las exigencias del mercado. $Y$ fue un buen debate que nos ha ayudado a recordar el riesgo que ya apuntaba Díez Hochleitner (2002, p.18) para el que el mundo universitario "parece rehuir o sustraerse ahora frecuentemente del principal papel que le corresponde en la formulación de una visión prospectiva y global de los problemas que atañen a la humanidad así como de la responsabilidad prioritaria en la búsqueda de las posibles soluciones". Esta observación nos parece muy relevante porque el reto actual es la promoción de los derechos de las personas para asegurar una vida digna y plena.

En nuestro caso, siempre hemos tenido presente que la universidad debe estar inmersa de forma activa en la sociedad de la que forma parte, y que su compromiso consiste en crear conocimiento a través de la investigación, pero también proponiendo, orientando y aplicando su saber en acciones concretas, decididamente comprometidas con la mejora de las condiciones de vida (Vilar, 2003, p.149). Creemos que la mejor forma de hacerlo es trabajando en tres grandes direcciones complementarias entre ellas: en primer lugar, formando buenos profesionales; en segundo lugar, participando en la construcción de respuestas ante los nuevos retos sociales; en tercer lugar, haciendo política, esto es, denunciando y creando opinión o ayudando a crearla sobre los grandes desafíos que se le presentan a la comunidad" (Vilar, 2003, p.159).

Son muchas y variadas las formas de asumir este compromiso. Desde nuestro punto de vista, hemos considerado que la mejor forma de hacerlo es mediante la formación de profesionales reflexivos, porque es un perfil profesional de carácter integrador que puede dar respuesta a los tres ejes que acabamos de plantear: comporta capacidad de comprensión y de análisis del 


Jesús Vilar Martín
Gisela Riberas Bargalló
Genoveva Rosa Gregori
Facultad de Educación Social y Trabajo Social Pere Tarrés
Universidad Ramon Llull
España

mundo complejo; implica rigor y capacidad para construir repuestas globales de carácter interdisciplinario, implementarlas y valorar sus efectos; finalmente, supone sentido moral y posicionamiento político de carácter crítico frente a las desigualdades y las injusticias del mundo actual. Tenemos el convencimiento que las características del mundo actual y las interacciones que en él se producen nos permiten afirmar que ética y complejidad van de la mano. Por ello, la formación debe desarrollar el sentido de servicio público, por lo que, para nosotros, la ética, entendida como el compromiso ante la sociedad del profesional, es un eje fundamental en la formación de profesionales reflexivos. Ahora bien, se trata de una concepción amplia y poliédrica del concepto: como formación filosófica que desarrolla la responsabilidad pública y el sentido moral, como capacitación técnica que exige rigor, eficacia y eficiencia técnica y como capacidad estratégica para gestionar situaciones injustas estresantes como puede ser la resolución de conflictos o dilemas morales (Riberas, G.; Vilar, J.; Rosa, G., 2014). En las páginas siguientes vamos a desarrollar de forma detallada las características del pensamiento reflexivo, su relación con el mundo complejo y las implicaciones en la formación.

\section{La complejidad, un signo de nuestro tiempo}

El mundo actual se caracteriza por el dinamismo y la complejidad. Así lo demuestran las aportaciones epistemológicas que se fueron presentando a lo largo del siglo XX (desde las propuestas de carácter sistémico de L.V. Bertalannfy, hasta las aportaciones actuales de Bauman y la modernidad líquida, pasando por las explicaciones sistémico-ecológicas de Bronfenbrenner o las ideas sobre pensamiento complejo de Morin). Estas aportaciones, diversas entre ellas tanto en sus enfoques teóricos como en su momento de aparición, fueron coincidiendo en algunas preocupaciones clave, como son "el desorden, el caos, la no-linealidad, el no-equilibro, la indecibilidad, la 
incertidumbre, la contradicción, el azar, la temporalidad, la emergencia, la autoorganización" (Rodríguez, L.; Leónidas, J.; 2011, p.148).

Todas estas "teorías de la complejidad"1, han puesto en evidencia las limitaciones que el pensamiento lineal y simplificador de carácter mecanicista y de orientación cartesiana tiene a la hora de entender la realidad y dar respuesta a las necesidades sociales contemporáneas, por lo que se han convertido en una alternativa epistemológica necesaria para entender las realidades actuales. Siguiendo a Amozorrutia, J.A. (2012, p.192) "enfrentar lo complejo implica una actitud que reconoce grados de aproximación a la cabal "comprensión/ explicación" de un fenómeno multidimensional, que por su dinámica particular, no se dejará atrapar por una conceptualización científica permanente. Lo complejo siempre será un detonador de la creatividad, porque solamente con creatividad e investigación interdisciplinaria es posible enfrentar el reto". Por otra parte, la perspectiva globalizadora del pensamiento complejo muestra las limitaciones en la comprensión de la realidad. Como indica Morin (2008, p.101), "la conciencia de la complejidad nos hace comprender que no podremos escapar jamás a la incertidumbre y que jamás podremos tener un saber total. (...) Estamos condenados a un pensamiento incierto".

Así pues, en el estudio de las realidades sociales habrá que integrar conocimiento y técnica, pero también los determinantes éticos y políticos de las relaciones sociales y los motivos que condicionan sus dinámicas: "el vínculo ciencia/técnica/sociedad/política es evidente. Es evidente, en estas condiciones, que la época en que los juicios de valor no tenían que interferir con la actividad científica está cerrada" (Morin, 2006:, p.77).

Así pues, sin renunciar a encontrar un cierto orden y claridad a la hora de entender la realidad, la incertidumbre de lo complejo nos hace ver con humildad la imposibilidad del control absoluto sobre ella, la provisionalidad de

\footnotetext{
${ }^{1}$ El origen de estas teorías es diverso y con enfoques complementarios (teoría de sistemas, cibernética, teoría de los fractales, teorías del caos, etc...), hasta el punto de poder hablar de "diversas generaciones de teorías de la complejidad" (Alhadeff-Jones, Michel, 2008, p.5-6). Para simplificar, este conglomerado de teorías se identifica de forma singular como "teoría de la complejidad", por lo que tienen en común todas ellas frente a los modelos mecanicistas.
} 


$$
\begin{gathered}
\text { Jesús Vilar Martín } \\
\text { Gisela Riberas Bargalló } \\
\text { Genoveva Rosa Gregori } \\
\text { Facultad de Educación Social y Trabajo Social Pere Tarrés } \\
\text { Universidad Ramon Llull } \\
\text { España }
\end{gathered}
$$

las interpretaciones y la caducidad de las propuestas de intervención. La concepción de la ciencia desde la complejidad acepta la imposibilidad del establecimiento de verdades. El camino desde este paradigma, que reconoce la subjetividad y la dimensión interpretativa de la realidad, es el de la incertidumbre, de lo desconocido, de lo imprevisible, (Peñalver, 1987, p.52).

Obviamente, la reflexión epistemológica tiene su reflejo en el terreno de la práctica profesional. Como hemos indicado en otros textos (Vilar, J.; Longás, J., 2013, p.67-68; Riberas, G.; Vilar, J.; Mora, P., p.31-33), nuestro punto de vista es que los modelos explicativos de la realidad de orientación mecanicista y sus correspondientes formas de actuación profesional de carácter tecnocrático no dan respuesta a los retos de la sociedad actual, dinámica y compleja. Las propuestas tecnocráticas funcionan en contextos asistenciales y de control más o menos sencillos. Comienzan a tener importantes limitaciones en un modelo de promoción de los derechos básicos y son insuficientes en las propuestas de promoción de las personas, que han de ser mucho más individualizadas, integrales en la singularidad de cada caso y contextualizadas en el territorio. Esta constatación es especialmente relevante porque los profesionales han tomado conciencia que trabajando desde esta perspectiva con frecuencia sus esfuerzos dan muy pobres resultados, lo que genera una gran sensación de malestar y decepción.

En la actualidad, las profesiones socioeducativas se encuentran inmersas en el paso de un modelo "actuador-aplicacionista", propio del paradigma cartesiano a un modelo "reflexivo-creador", que responde al paradigma de la complejidad. Mientras que el primero vive la novedad como un problema porque el supuesto del que parte es el de disponer de estrategias cerradas para solucionar problemas en una realidad predecible, el segundo vive la novedad y lo desconocido con naturalidad, porque el supuesto del que parte es que la realidad (los problemas y las soluciones que se les dan) siempre es una construcción que hay que elaborar a través de la investigación mediante la participación integrada de diversas disciplinas. El problema principal en estos momentos es haber tomado conciencia de las limitaciones de un modelo pero 


$$
\begin{gathered}
\text { Jesús Vilar Martín } \\
\text { Gisela Riberas Bargalló } \\
\text { Genoveva Rosa Gregori } \\
\text { Facultad de Educación Social y Trabajo Social Pere Tarrés } \\
\text { Universidad Ramon Llull } \\
\text { España }
\end{gathered}
$$

no tener suficientes elementos para dar el paso al segundo. Este paso no implica únicamente la modificación de las estrategias de intervención. Supone un cambio de perspectiva de carácter epistemológico que implica modificar la forma de entender la relación entre las disciplinas y, a su vez, la manera de interpretar la realidad y la forma de interactuar con ella.

\section{De la disciplinariedad a la transdisciplinariedad: cambios paradigmáticos}

Como es bien sabido, el enfoque más sencillo dentro de las ciencias para encarar la relación del conocimiento con la realidad es la disciplinariedad, entendida como la estructuración sistemática de un campo de saber determinado, con sus respectivas construcciones teóricas, metodologías de investigación y formas propias de interpretar los fenómenos que estudia. En un segundo momento podemos hablar de multidisciplinariedad, cuando las diferentes disciplinas analizan un mismo fenómeno, pero, aún reconociéndose entre ellas no hacen intercambios sistémicos; en el mejor de los casos, el análisis "multidisciplinar" se convierte en una suma de miradas que a veces se complementan, pero muchas otras se contradicen o interfieren entre ellas. En un tercer momento, hablamos de interdisciplinariedad cuando se crea un diálogo entre disciplinas de carácter integrador a la hora de leer la realidad. En este caso, se trataría de una situación cooperativa donde, sin renunciar a la esencia ni a las estructuras básicas de cada una de las disciplinas que intervienen en una situación, todas se refuerzan. Esto permite ofrecer miradas mucho más globales y útiles para el estudio de una complejidad organizada en referencia a aquellos fenómenos o problemas en donde intervienen un número amplio de factores o variables interrelacionadas que conforman un todo orgánico, que no resultan comprensibles por medio de enfoques reduccionistas-analíticos (Rodríguez, Aguirre; 2011, p.152).

Pero la complejidad de lo real supera las posibilidades de la interdisciplinariedad, por lo que el último paso es crear una mirada que sea capaz de trascender la perspectiva de las disciplinas con el objetivo de 


$$
\begin{gathered}
\text { Jesús Vilar Martín } \\
\text { Gisela Riberas Bargalló } \\
\text { Genoveva Rosa Gregori } \\
\text { Facultad de Educación Social y Trabajo Social Pere Tarrés } \\
\text { Universidad Ramon Llull } \\
\text { España }
\end{gathered}
$$

responder de manera más eficiente y eficaz a la realidad compleja de los fenómenos estudiados. Aparece aquí la transdisciplinariedad.

Como indica Nicolescu (1998, p.37), el impulsor más destacado de este concepto en su Manifiesto de la Transdisciplinariedad, "la transdisciplinariedad busca la comprehensión del mundo presente en el cual uno de los imperativos es la unidad del conocimiento. (...) La disciplinariedad, la pluridisciplinariedad, la interdisciplinariedad y la transdisciplinariedad son las cuatro flechas de un solo y mismo arco: el del conocimiento".

Se trata de una nueva perspectiva (y, sobretodo, de una nueva actitud) que busca la comprensión global de una situación, más allá de las constricciones que cada disciplina impone en la aproximación al estudio de la realidad. Comporta superar las fronteras clásicas de conocimiento y en este sentido adopta un enfoque ecléctico y constructivo, de carácter contextualizador.

Como indican Carrizo, L.; Espina, M.; Klein, J, (2004, p. 17), "Lo importante es que estos posicionamientos coinciden en que esa nueva etapa tiene como contenido central una reconstrucción en el plano epistemológico transdisciplinar, que intenta recuperar una visión compleja de lo social".

Es evidente que los profesionales que trabajan en el terreno socioeducativo se encuentran cada día con situaciones que requieren esta mirada transdisciplinar. La Interdisciplinariedad se muestra insuficiente para explicar y ofrecer alternativas a las problemáticas con las que deben lidiar, que integran aspectos psicológicos, interpsicológicos, emocionales, económicos, sociales culturales y, en muchos casos, también de salud. Por todo ello, nos parece un planteamiento que conviene tener en cuenta a la hora de pensar en la capacitación de profesionales reflexivos que trabajarán en entornos complejos. Tal como lo entendemos, ésta es la única forma de responder al reto que Delors planteó en 1996 con los "cuatro pilares de la educación" como elementos centrales frente a un futuro incierto y cambiante: aprender a conocer, aprender a hacer, aprender a vivir juntos y aprender a ser. 
Repercusiones de la transprofesionalidad en la formación de profesionales reflexivos.

Alineados con los planteamietos ya clásicos sobre la reflexión desde la acción apuntada por Schön (1992), la investigació-acción (Stenhouse, 1987; Elliot, 1989), y la educación crítica (Gimeno Sacristán, 1987; Carr, Kemmis, 1988), consideramos imprescindible que la universidad siempre ha de estar dirigida a formar profesionales reflexivos, esto es, personas que están capacitadas técnicamente y a la vez críticamente para solucionar problemas concretos e inmediatos de su práctica profesional cuando esto sea posible, pero que también tienen conocimientos extensos para entender su profesión y la forma como ésta interactúa con las demás desde una perspectiva global. Este conjunto de competencias les ha de permitir disponer de un amplio margen para tomar decisiones porque lleva implícita la actividad investigadora frente a situaciones complejas (Riberas, G.; Vilar, J., Mora, 2013, p.35). En este caso, se supera la oposición entre teoría y práctica, que se convierte en complementariedad entre reflexión y acción.

La formación de profesionales reflexivos implica llevar el debate de la transdisciplinariedad a la forma de organizar los currículums y los planes de estudio. Esto supone importantes dificultades a la hora de implementarlo en la universidad, por la tendencia conservadora a organizar el conocimiento y entender la formación. Con demasiada frecuencia, éstos están centrados y organizados desde la lógica interna de las disciplinas y el saber del profesor/a. Como nos recuerda Cortina (2003, p.14), "la realidad tiene problemas pero la universidad tiene departamentos". Si la organización actual separa en forma arbórea las diferentes parcelas de conocimiento, la transcisciplinariedad se caracteriza por ser "el arte de descubrir puentes entre diferentes objetos y áreas de conocimiento (Carrizo, L.; Espina, M.; Klein, J, 2004, p.32)”. Se trata de encontrar mecanismos que faciliten la comunicación fluida entre disciplinas. Aunque en teoría las ventajas de este planteamiento resultan evidentes, los mismos autores (2004, p.60-61), nos indican algunos de los principales obstáculos en su implementación: 


$$
\begin{gathered}
\text { Jesús Vilar Martín } \\
\text { Gisela Riberas Bargalló } \\
\text { Genoveva Rosa Gregori } \\
\text { Facultad de Educación Social y Trabajo Social Pere Tarrés } \\
\text { Universidad Ramon Llull } \\
\text { España }
\end{gathered}
$$

- Epistemológicos, referidos a la tensión entre paradigmas de conocimiento.

- Culturales, referidos a las brechas entre la cultura científica, humanista y popular.

- Institucionales, referidos a la defensa de territorios de saber/poder en las Universidades, Facultades o Colegios Profesionales.

- Organizacionales, referidos a los instrumentos tecnocráticos de las reformas universitarias: estructuras de los programas, tipo de evaluación, relación entre campos de saber, etc.

- Psicosociales, referidos a la crisis y transformación de las identidades profesionales.

- Económicos, referidos por un lado a las características de un mercado de empleo tecnocrático e hiperespecializado y, por otro, a las fuentes de financiamiento para la investigación y el desarrollo transdisciplinarios.

Como puede verse, el gran problema de la universidad no está tanto en la producción de conocimiento de las diferentes disciplinas (condicionado, eso sí, por los presupuestos de que se dispongan), como en la trasferencia de esos conocimientos a la sociedad de la que forma parte, en este caso, mediante la formación. La "Agència de qualitat del sistema universitari de Catalunya" (AQU), nos recuerda que, aunque hay un consenso explícito respecto que la investigación y la docencia son los dos ejes principales de la universidad, se constata la falta de interacción entre la función docente y la función investigadora, y que el currículum que se imparte tiene poco que ver con el nivel de especialización de la investigación en cada una de las disciplinas (AQU, 2005, p.12). Los cambios principales que habrá que plantear se centran en conseguir que la investigación traspase la clasificación tradicional por materias y plantee miradas compartidas a los fenómenos complejos que estudia. Además, que el currículum formativo supere la división clásica de las disciplinas y sus materias dentro de un currículum. El currículum, como forma de orden y de estructura debe existir, pero la metodología de formación debe asegurar que se superan las limitaciones de la ordenación curricular. Las propuestas curriculares han de centrar sus esfuerzos en el diseño de metodologías que aseguren la comunicación fluida entre áreas de conocimiento. Esto implica pensar el proceso y las secuencias de enseñanza- 


$$
\begin{gathered}
\text { Jesús Vilar Martín } \\
\text { Gisela Riberas Bargalló } \\
\text { Genoveva Rosa Gregori } \\
\text { Facultad de Educación Social y Trabajo Social Pere Tarrés } \\
\text { Universidad Ramon Llull } \\
\text { España }
\end{gathered}
$$

aprendizaje y no sólo el contenido que se debe enseñar. La materia, aun siendo fundamental, ha de quedar en un plano similar al del procedimiento de aprendizaje y a la conciencia que el estudiante debe tener sobre ese proceso. Hay que evitar lo que Meirieu (1988, p. 69) describe de la siguiente manera: "Las disciplinas se han convertido, en el curso del tiempo, sin ni siquiera darse cuenta quienes han presidido su organización, en (...) jirones de conocimientos extirpados de tratados eruditos y compilados en manuales. Ya no habita en ellas lo que podría de veras darles vida, el interrogante fundacional que permitiría a seres que entran en el mundo hacérselas propias y acrecentarlas".

Generar nuevamente este "interrogante fundacional" exige un cambio en la forma de entender la docencia y plantear un nuevo estilo de trabajo. Para empezar, aunque parezca una obviedad, el profesor/a ha de entender que es un docente que anima situaciones de enseñanza-aprendizaje, no únicamente un investigador que expone sus conocimientos. La persona más experta en cualquier área ha de comprender que en el momento que imparte una clase se convierte en docente y que el saber experto de su área no es garantía suficiente para asegurar la formación de buenos profesionales. Por eso es de vital importancia la reflexión pedagógica en la formación universitaria.

Aunque se sigan impartiendo clases magistrales, hay que diversificar les metodologías hacia otras experiencias más participativas y activas. El docente ha de dejar de ser el trasmisor de conocimiento para convertirse en un creador de situaciones de aprendizaje (AQU, 2005, p.17) donde la reflexión crítica sea el entorno natural de trabajo y como mínimo suponga: trabajar con la incertidumbre; desarrollar la autoconciencia y la metacognición, de manera que el estudiante tenga conciencia de su proceso de aprendizaje (lo que aprende, cómo lo aprende y lo que queda por aprender); diferenciar las conjeturas de la observación sistemática; inferir ideas a partir de datos o utilizar el conocimiento abstracto en situaciones concretas y próximas (Bain, 2006, p.96-97).

Como puede verse, el problema no es qué enseñar, sino cómo hacerlo para que el estudiante lo integre de forma significativa, le resulte útil para una aproximación al mundo complejo con el que se encontrará y realmente se 


$$
\begin{gathered}
\text { Jesús Vilar Martín } \\
\text { Gisela Riberas Bargalló } \\
\text { Genoveva Rosa Gregori } \\
\text { Facultad de Educación Social y Trabajo Social Pere Tarrés } \\
\text { Universidad Ramon Llull } \\
\text { España }
\end{gathered}
$$

convierta en un profesional reflexivo creador de conocimiento y no únicamente un consumidor de conocimiento que han elaborado otros (Riberas, G.; Vilar, J.; Mora, P., 2013, p.34). Por esta razón, el proceso formativo ha de convertirse en un proceso de investigación donde el estudiante, mediante experiencias investigadoras de aprendizaje, aprende doblemente: por un lado los contenidos de las disciplinas pero, a la vez, las competencias que permiten hacer un uso integrado de las mismas en la aproximación a la realidad social (AQU, p.21). Esto no significa que todos los estudiantes deban ser investigadores, pero sí que la experiencia de investigación como metodología de formación les permitirá desarrollar una actitud investigadora en su práctica profesional cotidiana. En definitiva, hay que substituir la dicotomía entre investigación y docencia y experimentarlas de forma complementaria en la experiencia formativa (Bain, 2006, p.188). En consecuencia, es necesario desarrollar una línea estratégica que impulse un cambio en las metodologías formativas en la universidad. La Secretaría de estado de universidades e investigación (2006, p.135) destacaba la necesidad de crear proyectos específicos para impulsar este cambio. En concreto, proponía la creación de un Plan Específico para la Renovación de las Metodologías Educativas (PERME), con diferentes acciones: impulso y ejecución de proyectos de renovación, formación del profesorado y difusión y evaluación de resultados.

Los diferentes argumentos que hemos expuesto hasta este momento sobre complejidad encuentran una correlación con las propuestas formativas que venimos desarrollando desde el año 1992, cuando iniciamos los estudios de Educación Social. Se trata de utilizar metodologías que permitan llevar a la formación los preceptos teóricos sobre el pensamiento complejo y la profesionalidad reflexiva y que finalmente, permita su evaluación. Esto requiere reflexionar sobre cuál ha de ser el resultado del aprendizaje y cómo debe llevarse a cabo. Queremos hacer notar que hablamos de "resultado de aprendizaje" y no de "contenidos aprendidos" porque la profesionalidad reflexiva exige integraciones significativas de elementos que van más allá de los contenidos clásicos. Como es bien sabido, el EEES propone el concepto de 


$$
\begin{gathered}
\text { Jesús Vilar Martín } \\
\text { Gisela Riberas Bargalló } \\
\text { Genoveva Rosa Gregori } \\
\text { Facultad de Educación Social y Trabajo Social Pere Tarrés } \\
\text { Universidad Ramon Llull } \\
\text { España }
\end{gathered}
$$

competencia como elemento aglutinador de todos estos elementos. La discusión sobre este concepto y las dificultades que las diferentes titulaciones han tenido para concretarlas sería motivo de un artículo en sí mismo. En este caso, vamos a entender las competencias como un constructo que movilizan diferentes recursos personales que permiten integrar conocimientos, estrategias y actitudes. Esto implica saber y disponer de conocimientos, saberlos utilizar en situaciones complejas de forma rigurosa, manifestar actitudes críticas y responsables frente a la realidad y el propio trabajo y ser conscientes del propio proceso reflexivo.

Como los referentes teóricos para definirlas son múltiples, ya vengan de la universidad y personas expertas, las administraciones educativas 0 las asociaciones profesionales, en este caso, indicaremos las familias de competencias en las que coinciden la mayoría de textos ${ }^{2}$ y que, para nosotros, son relevantes para la formación en el perfil profesional que venimos describiendo: competencias relacionadas con la integración de conocimientos y habilidades frente a situaciones complejas; competencias relacionadas con la capacidad de investigación y análisis; competencias que hacen referencia al autoconocimiento y la metacognición respecto del propio proceso reflexivo; competencias para aproximarse a los contextos sociales, desde una perspectiva transversal; competencias que movilizan la capacidad de construir conjuntamente, implicarse en los equipos y generar conocimiento; competencias para el abordaje de situaciones críticas (crisis, dilemas...). Las estrategias y metodologías formativas para abordar estas competencias son múltiples y diversas. Entre ellas, destacamos las siguientes: seguimiento tutorial, cuestionarios autoevaluativos, investigación aplicada, transversalidad entre asignaturas, aprendizaje basado en problemas y casos, seminarios cooperativos, habilidades sociales o análisis y gestión de dilemas éticos.

\footnotetext{
${ }^{2}$ En este caso, como referentes teóricos seguimos a Morin (1999), Perrenoud (2004), Imbernon (2007) o Zabala (2007).
} 


\section{La contextualización de las capacidades competenciales: territorio redes}

Para acabar esta exposición, dedicaremos las últimas líneas a dibujar a grandes rasgos dos elementos centrales en la formación de profesionales reflexivos: la importancia del territorio como espacio de contextualización de las competencias (siguiendo la máxima de la ecología "piensa global, actúa local") y las redes como estructuras de trabajo eficaces y eficientes para la construcción de conocimiento ante las necesidades de ese territorio. La calidad de estas estructuras mostrará hasta qué punto se han adquirido las competencias del profesional reflexivo.

El territorio no es sólo el espacio físico donde vive una población y se distribuyen los servicios. Es, fundamentalmente, un lugar simbólico donde se producen las interacciones entre personas, donde emergen las dificultades y donde se crean las respuestas posibles (Vilar, 2008, p.272). Implica disponer de un lugar relacional por lo que, como indica Augé, (2006, p.57), se trata de un lugar antropológico donde coinciden unos rasgos identificatorios, relacionales e históricos. En este sentido, se convierte en el mejor ejemplo de situación compleja a la que deberá atender el profesional. El territorio determina la naturaleza de las problemáticas y también las posibilidades de construir respuestas. La realidad sistémica y compleja de las problemáticas sociales requiere que los distintos focos de saber o agentes socioeducativos que en él confluyen trabajen de forma interactiva en forma de redes para crear respuestas realmente efectivas y sólidas.

Un aspecto que debemos repasar aunque sea brevemente es la calidad de las estructuras de red. En la actualidad se está haciendo un uso polisémico del concepto que comporta mucha confusión, hasta el punto que las prácticas profesionales que van más allá del trabajo en solitario a menudo ya se las define como red (Díaz, J.; Civis, M., 2011, p.416).

De la misma forma que las disciplinas, como vimos anteriormente, han ido progresando desde un trabajo aislado hasta una cooperación transdisciplinaria, el mismo proceso ha desarrollado el trabajo interprofesional. La estructura más 


$$
\begin{gathered}
\text { Jesús Vilar Martín } \\
\text { Gisela Riberas Bargalló } \\
\text { Genoveva Rosa Gregori } \\
\text { Facultad de Educación Social y Trabajo Social Pere Tarrés } \\
\text { Universidad Ramon Llull } \\
\text { España }
\end{gathered}
$$

arcaica de trabajo en el territorio es la ausencia de comunicación entre agentes que se ignoran entre ellos, lo que a menudo comporta duplicidades y a menudo interferencias o contradicciones entre ellos; este primer momento se corresponde con la disciplinariedad. Una segunda forma algo más elaborada, pero aún simple, es la coordinación entre agentes que se comunican entre ellos para evitar las duplicidades o interferencias del momento anterior, pero sin haber trabajo cooperativo; este momento se correspondería con la multidisciplinariedad. La tercera forma de relación cualitativamente diferente, es cuando se produce cooperación entre agentes, lo que implica un cierto nivel de interdependencia que puede ser alto, pero sin perder de vista la matriz disciplinaria o el marco profesional de cada uno de ellos; en este caso, podríamos hablar de interdisciplinariedad. Finalmente, el momento más elaborado sería el de construcción conjunta entre agentes que elaboran una única respuesta que trasciende la referencia profesional y disciplinaria; este momento, menos frecuente, se correspondería con la transprofesionalidad (Vilar, 2008, p.273).

Si tenemos en cuenta los aspectos que acabamos de comentar, es imprescindible definir los criterios mínimos que permitan afirmar que una red realmente lo es y responde a criterios de transprofesionalidad donde se ponen en juego competencias reflexivas. Una red que realmente cumple su función es aquella que no sólo proyecta un trabajo integrado entre profesionales sino que, además, genera capital social en el territorio al incorporar el máximo de agentes informales de forma corresponsable (Díaz, J.; Civis, M., 2011, p.425). Siguiendo los mismos autores, la red que genera capital social es aquella que presenta las siguientes características: proximidad entre agentes y problemáticas abordadas; transversalidad entre servicios y profesionales, de manera que el centro de la acción no sea la estructura profesional sino la persona atendida; horizontalidad entre participantes y eliminación de relaciones jerárquicas, aunque puedan darse asimetrías en las responsabilidades que cada elemento de la red asume; cooperación entre los participantes, en tanto que actitud voluntariosa para construir conjuntamente; corresponsabilidad e 


$$
\begin{gathered}
\text { Jesús Vilar Martín } \\
\text { Gisela Riberas Bargalló } \\
\text { Genoveva Rosa Gregori } \\
\text { Facultad de Educación Social y Trabajo Social Pere Tarrés } \\
\text { Universidad Ramon Llull } \\
\text { España }
\end{gathered}
$$

implicación en la construcción de respuestas; proactividad y proyección, como formas anticipatorias del sistema que supera estructuras meramente reactivas. Como puede verse, la red es un sistema que aprende, por lo que en cada caso habrá que ver su complejidad y su riqueza, en función de los elementos que la componen porque la calidad de las respuestas que se construyan dependerá de esta riqueza y complejidad (Vilar, 2008, p.269). La red que se construye no puede desvincularse del territorio; los agentes que la configuran también son parte del mismo, de manera que, más allá de los aspectos puramente organizativos o de contenido de la red, cobra especial importancia la perspectiva relacional y, en consecuencia, la dimensión ética y emocional.

No hay que perder de vista que lo que da sentido a la construcción de conocimiento, la formación de profesionales reflexivos y la generación de estructuras eficientes ante la complejidad es la construcción de territorios donde sea posible vivir humanamente. Contribuir a ello ha sido el objetivo de este artículo.

Resumo: Este artículo expõe que uma das responsabilidades da universidade neste momento é a formação de profissionais reflexivos e críticos que possam trabalhar em ambientes de complexidade. Descrevem-se as características dos ambientes complexos e a continuação se expõem as repercussões de esse enfoque no modo de desenhar a formação de profissionais. Finalmente se descreve as redes socioeducativas como formar de concretizar as estratégias de trabalho em um território.

Palavras chaves: pensamento complexo, profissional reflexivo, compromisso.

\section{Bibliografia}

ALHADEFF-JONES, M. Trois Générations de Théories de la Complexité: Nuances et Ambiguités. 2008. Disponible en http://www.intelligencecomplexite.org/fileadmin/docs/0805michel. Consultado el 17-3- 2014.

AMOZORRUTIA, J.A. Complejidad y ciencias sociales. Un modelo adaptativo para la investigación interdisciplinaria. México: Universidad Nacional Autónoma de México. Centro de Investigaciones Interdisciplinarias en Ciencias y Humanidades, 2012. 


Jesús Vilar Martín
Gisela Riberas Bargalló
Genoveva Rosa Gregori
Facultad de Educación Social y Trabajo Social Pere Tarrés
Universidad Ramon Llull
España

OLIVERAS, J. (Coord.). Marc general per a l'avaluació de la interacció entre la recerca i la docència a la universitat. Barcelona: AQU, 2005. Disponible en http://www.aqu.cat/doc/doc_69247575_1.pdf

AUGÉ, M. Los no lugares. Espacios del anonimato. Barcelona: Gedisa, 2006.

BAIN, K. El que fan els millors professors universitaris. València: Publicacions de la Universitat de València, 2006.

BRICALL, J.M. Universidad 2000. Madrid: Conferencia de Rectores de las Universidades Españolas, 2000.

CARR, W.; KEMMIS. S. Teoria crítica de la enseñanza. Barcelona: Martínez Roca, 1988.

CARRIZO, L.; ESPINA, M.; KLEIN, J. Transdisciplinariedad y Complejidad en el Análisis Social. Documentos de debate MOST. UNESCO: París, 2004. Disponible en http://unesdoc.unesco.org/images/0013/001363/136367s.pdf

DELORS, J. Educació: hi ha un tresor amagat a dins. Barcelona: Centre UNESCO de Catalunya, 1996.

DÍAZ, J., CIVIS, M. Redes Socioeducativas promotoras de capital social en la comunidad: un marco teórico de referencia. Cultura y Educación, Madrid, n. 23 (3), p. 415-429, 2011.

DÍEZ, R. ¿Hacia donde va la Universidad? Crítica, Madrid, n. 896, Junio 2002.

ORTEGA Y GASSET Y Gasset, J. Misión de la Universidad. Madrid: Revista de Occidente en Alianza Editorial, 1982.

GIMENO, J. Teoría de la enseñanza y desarrollo del currículo. Madrid: Anaya, 1987.

IMBERNON, F. La formación permanente del profesorado. Nuevas ideas para formar en la innovación y el cambio. Barcelona: Graó, 2007.

MEIRIEU, Ph. Frankenstein Educador. Barcelona. Laertes, 1998. 
MORIN, E. Los siete saberes necesarios para la educación del futuro. París: Unesco, 2003.

MORIN, E. El método. V.6 Ética. Madrid: Cátedra, 2006.

MORIN, E. Introducción al pensamiento complejo. Barcelona: Gedisa, 2008.

NICOLESCU, B. La transdisciplinariedad. Manifiesto. París: du Rocher, 1996. Disponible en http://www.ceuarkos.com/manifiesto.pdf

ELLIOT, J. Pràctica, recerca i teoria en educació. Vic: Eumo Editorial, 1989.

PEÑALVER, C. Las explicaciones sistémicas: una aproximación a la epistemología sistémica. En CASTILLEJO, J.L.; COLOM, A. Pedagogía sistèmica. Barcelona: Ceac, 1987. p. 45-63.

PERRENOUD, Ph. Diez nuevas competencias para enseñar. Barcelona: Graó, 2004.

RIBERAS, G.; VILAR, J.; MORA, P. Elaboració de projectes socials. Disseny i avaluació de les accions socioeducatives. Barcelona: Claret, 2013.

RIBERAS, G.; VILAR, J.; Rosa, G. La ética en los grados de Educación Social y trabajo Social. Una mirada prospectiva. En SEECI, 2014 (en prensa).

RODRÍGUEZ, L.; LEÓNIDAS, J.; (2011). Teorías de la complejidad y ciencias sociales. Nuevas estrategias epistemológicas y metodológicas. Nómadas.

Revista Crítica de Ciencias Sociales y Jurídicas, 30, n. 2, 2011. p. 147-166.

Disponible en

http://pendientedemigracion.ucm.es/info/nomadas/30/rdzzoya_aguirre.pdf

SECRETARÍA DE ESTADO DE UNIVERSIDADES E INVESTIGACIÓN. Propuestas para la renovación de las metodologías educativas en la universidad. Madrid: Ministerio de educación y ciencia, 2006. Disponible en http://www.catedraunesco.es/archivos/metodologias.pdf 
SCHÖN, D. A. La formación de profesionales reflexivos. Hacia un nuevo diseño de la enseñanza y el aprendizaje de las profesiones. Barcelona: Paidós, 1992.

STENHOUSE, L. La investigación como base de la enseñanza. Madrid: Morata, 1987.

UNESCO. Declaración mundial sobre la Educación Superior en el siglo XXI: visión y acción. París: UNESCO, 1998. Disponible en http://unesdoc.unesco.org/images/0011/001163/116345s.pdf

VILAR, J. Responsabilidad y compromiso ético de los formadores en pedagogía social y educación social. Pedagogía social: revista interuniversitaria, $9, \quad 2003 . \quad \mathrm{p}$ 143-160. Disponible en http://www.redalyc.org/articulo.oa?id=135018332008

VILAR, J. (2008). Implicaciones éticas del trabajo en red y la acción comunitaria. Cultura y Educación, Madrid, 20, 2008. p. 266-277.

VILAR, J.; LONGÁS, J. Adolescentes en Riesgo o Especial Vulnerabilidad. Buenas Prácticas Socioeducativas desde y con el Sistema Educativo en Cataluña. En SANTIBÁÑEZ, R. y MARTíNEZ-PAMPLIEGA, A. (Coords.) Intervención comunitaria con adolescentes y familias en riesgo. Barcelona: Graó, 2013. p. 59-74.

ZABALA, A.; ARNAU, L. Cómo aprender y enseñar competencias. Graó: Barcelona, (2007).

* Recebido em: 09 de abril de 2014.

* Aprovado em: 30 maio de 2014. 